Mots. Les langages du politique

Proximité

\title{
Du patrimoine au « proximoine » ou le présent d'une illusion
}

Jean-Michel Utard

\section{(2) OpenEdition}

Journals

Édition électronique

URL : https://journals.openedition.org/mots/223

DOI : $10.4000 /$ mots. 223

ISSN : 1960-6001

Éditeur

ENS Éditions

Édition imprimée

Date de publication : 1 mars 2005

Pagination : 137-144

ISBN : 2-84788-077-1

ISSN : 0243-6450

\section{Référence électronique}

Jean-Michel Utard, « Du patrimoine au « proximoine » ou le présent d'une illusion », Mots. Les langages du politique [En ligne], 77 | 2005, mis en ligne le 31 janvier 2008, consulté le 23 avril 2022. URL : http:// journals.openedition.org/mots/223; DOI : https://doi.org/10.4000/mots.223 


\section{Du patrimoine au "proximoine» ou le présent d'une illusion}

Quelle différence y a-t-il entre un monument et un monstre ? Leur terminaison, car les deux mots dérivent du latin moneo qui signifie «faire penser à quelque chose». L'un et l'autre objet sont donc des signes, c'est-à-dire une présence attestant une absence. Mais l'un est tourné vers le passé et sert de support à la mémoire : le monument, c'est ce qui nous fait nous souvenir. Alors que l'autre est dirigé vers l'avenir : le monstre nous avertit d'un malheur à venir (par opposition à l'ange, son double inverse, qui vient annoncer des jours radieux). Mais, qu'il s'agisse d'un bâtiment ou d'un signal avertisseur, il faut bien que quelqu'un nous ait «fait» ce signe. Cependant, si l'un est le fruit de la geste humaine, l'autre est créé par un geste des dieux. Le monstre conserve alors une signification éternelle, hors de l'espace et du temps, même si les métamorphoses de son apparence ne nous permettent pas toujours de retrouver ce sens. Le monument au contraire, dans la permanence de sa forme (conservée ou restaurée), change de sens au gré du regard des hommes : du parpaing au témoin, de l'indice au symbole, de l'identité à l'universalité.

L'activité législative intense sur la décentralisation de l'État n'épargne pas ces témoins d'une histoire instituée. Au contraire même, par une sorte de paradoxe temporel, le transfert aux collectivités territoriales de la gestion du patrimoine monumental semble revêtir un caractère d'urgence que contredit sa stabilité immobilière. Sans doute parce qu'il est plus facile d'argumenter pour légitimer la gestion de proximité des monuments, des bâtiments scolaires et des infrastructures de transports que celle des hommes, même si celle des uns entraine celle des autres. Mais un monument historique n'est pas un tas de cailloux, c'est une construction symbolique, et sa relocalisation équivaut à une réécriture de l'histoire. Dans certains cas, l'hybridation du terroir et de l'histoire risque de produire des monuments symboliquement modifiés, voire des monstres symboliques.

Université Strasbourg 3, CRAPE-CNRS, jm.utard@wanadoo.fr 


\section{La décentralisation : prope versus longe}

Cette politique de décentralisation, entreprise depuis plus de vingt ans, s'effectue désormais sous le signe de la «proximité». Dans sa déclaration de politique générale de juillet 2002, le Premier ministre a placé son action dans la perspective d'une «République des proximités». Celle-ci est présentée comme une attente des citoyens, une exigence, voire un besoin. Elle est en même temps un principe et une réalité spatiale. Le principe est celui de la simplification administrative, c'est-à-dire la fin du face à face permanent du citoyen avec l'État au nom du principe de subsidiarité, et de la transparence, c'est-à-dire la suppression des médiations au profit d'un lien direct avec les responsables de l'action publique à tous les niveaux. La proximité est aussi une réalité spatiale, dans la définition qu'en donne Jean-Pierre Raffarin lui-même, comme «champ d'action des départements, des communes et de leurs regroupements ${ }^{1}$, le niveau régional étant alors celui de la cohérence et le niveau étatique celui de la définition des "grands principes» et de la régulation des équilibres interrégionaux.

Une loi constitutionnelle relative à l'organisation décentralisée de la République a été adoptée le 17 mars 2003 par le Parlement réuni en congrès au château de Versailles, haut lieu symbolique du patrimoine historique de la France. Dans l'exposé des motifs accompagnant le projet de loi lors de son dépôt devant les Assemblées, il est rappelé l'attachement des Français aux principes fondateurs de la République que sont «l'indivisibilité du territoire et l'égalité des citoyens devant la loi». Répondant par avance aux craintes de les voir remis en cause par les mesures de décentralisation, le texte affirme que «l'idée selon laquelle ces principes exigeraient que l'on bride les initiatives locales appartient, en revanche, au passé». De même que la centralisation ne permet d'éviter ni les disparités, ni les inégalités, de même la décentralisation n'empêche ni la cohérence ni les solidarités. Quelque chose, donc, aurait changé, qui permettrait aujourd'hui de procéder au mouvement inverse de celui qui a permis l'émancipation des hommes des dépendances locales et des références identitaires.

Force est de constater que le socle argumentatif est bien fragile. La croyance dans les vertus de la proximité relève d'une solide évidence à laquelle la raison a du mal à s'opposer : tout ce qui est plus près est proche. Et réciproquement. Il ne s'agit pas ici de nier la nécessité de donner plus d'autonomie aux individus et aux instances infra-étatiques dans une démocratie moderne. Mais l'acti-

1. Déclaration de politique générale, Assemblée nationale, 3 juillet 2002. 
vité parlementaire semble pour l'instant plus hantée par le souci d'alléger le poids de l'État que de favoriser le fonctionnement de la démocratie locale. Et les conflits de territoires qui se manifestent dans les débats des Assemblées laissent mal augurer des solidarités locales. De même que la conciliation de la cohérence nationale et de la stratification des territoires risque de conduire à un «empilement des structures et objets administratifs non identifiés», pour reprendre les termes du Premier ministre ${ }^{2}$, mais cette fois au niveau des collectivités territoriales. Le cas particulier du patrimoine historique, et plus spécifiquement des monuments nationaux, révèle les contradictions que masque la référence incantatoire à la proximité.

\section{Patrimoine et territoire : propior versus longius}

Le patrimoine, c'est d'abord l'ensemble des biens que nous héritons de nos "pères», qu'en principe nous préservons et même faisons fructifier pour les transmettre si possible accrus à nos descendants. Au cours du processus, des transformations sont possibles : on peut fondre les couverts, vendre les meubles, transformer les immeubles, etc., pour tenir compte de l'évolution de la conjoncture et du contexte, pourvu que la «valeur» des biens reste au moins constante. Le contrexemple étant celui du fils prodigue.

Mais ce que ce modèle économique de simple accumulation occulte, ou plutôt absorbe dans la valorisation marchande, c'est la dimension symbolique du patrimoine. Un château ou plus modestement une demeure, un meuble ou un bijou sont toujours peu ou prou «de famille». C'est dire assez le lien fort qui unit le patrimoine et les références identitaires, lien renforcé dans le cas du patrimoine bâti par l'attachement au territoire.

C'est par une démarche similaire d'association entre un support matériel et sa valeur d'identification qu'a été constitué le patrimoine national. «De la cathédrale à la petite cuillère » (Malraux), tous les objets inventoriés sont autant de nœuds dans le réseau qui constitue la toile de notre mémoire commune. Mais à l'inverse de la transmission des biens privés, c'est par un geste d'arrachement à la lignée et au terroir qu'il peut se constituer comme référent identitaire collectif. Et sa valeur économique ne lui vient qu'après l'attribution d'une valeur patrimoniale symbolique. Ces valeurs enfin ne peuvent durer dans le temps que par une permanence de la forme de l'objet : l'armoire $18^{\mathrm{e}}$ transformée en poulailler ne peut prétendre à la préservation patrimoniale qu'au prix d'une restauration rigoureuse.

2. Déclaration aux Assises des libertés locales organisées le vendredi 25 octobre 2002 à Marseille. 
Cette «socialisation» du patrimoine pose évidemment la question de la légitimité de l'instance habilitée à produire du symbole et à en assurer la gestion. Suivant quelle procédure va-t-on partir à la découverte des objets susceptibles de patrimonialisation ? D’après quels critères va-t-on décider qu'ils relèvent de la juridiction commune ? Suivant quelle histoire va-t-on décider des formes de leur protection ? Quelle logique va présider à l'allocation des ressources nécessaires à leur conservation ? Autant de questions dont les réponses différenciées correspondent aux choix politiques que chaque pays a faits et aux dispositifs administratifs qu'il a imaginés.

En France, la gestion du patrimoine relève de la responsabilité du ministère de la Culture auquel sont confiées les missions de l'inventorier, le conserver et le valoriser. Leur mise en œuvre a lieu au sein des directions régionales des affaires culturelles (DRAC) et des services départementaux de l'architecture et du patrimoine (SDAP), qui sont des services déconcentrés de l'État. Les conservateurs, responsables des services de l'inventaire, des monuments historiques et de l'archéologie, et les architectes des bâtiments de France (ABF) y sont des médiateurs. D'un côté, ils garantissent la cohérence de la définition et de la conservation des biens patrimoniaux par la référence à des principes scientifiquement et législativement établis au niveau national; de l'autre, ils en assurent la nécessaire adaptation aux réalités du terrain et aux dynamiques des acteurs locaux. Autorisations et crédits, autrement dit bâtons et carottes sont les instruments de leur travail de régulation.

L'argumentation en faveur d'une gestion de proximité du patrimoine historique s'appuie sur les dysfonctionnements évidents que produit une accumulation règlementaire et organisationnelle depuis plus d'un siècle et demi, sur le sentiment d'arbitraire que provoquent certains arbitrages, sur le décalage entre les dynamiques locales et les contraintes de la conservation. Le champ du patrimoine n'a cessé de s'étendre : du bâtiment à ses abords, du monument aux ensembles urbains, des sites historiques aux espaces industriels, etc. Autant dire que des contradictions émergent entre les contraintes de la protection et les exigences du développement, entre les couts marginaux qu'engendre la conservation et la faiblesse des ressources que génère le tourisme patrimonial, entre le respect des formes léguées par l'histoire et l'évolution du gout architectural.

Un ensemble de textes de réflexion s’inspirant du rapport de la commission «Patrimoine et décentralisation ${ }^{3}$ contribue à dessiner ce que pourrait être une gestion décentralisée. Pour les monuments dont il reste propriétaire, l’État conserverait la totalité des compétences. Pour le reste du patrimoine, les com-

3. Rapport de la commission «Patrimoine et décentralisation» au ministre de la Culture et de la Communication. «Réflexions et propositions pour une politique nationale du patrimoine (État, collectivités territoriales et secteur privé)», président : J.-P. Bady, novembre 2002. 
pétences se distribueraient entre les différents niveaux territoriaux. L'État conserverait ses prérogatives en matière de contrôle scientifique et technique et resterait garant de la cohérence scientifique nationale (doctrine de protection, outils méthodologiques de l'inventaire, accès des citoyens à l'information). La protection et la maitrise d'ouvrage seraient confiées à la compétence de la région, ce territoire étant «suffisamment vaste pour permettre des comparaisons significatives entre édifices protégés et à protéger et appliquer des critères homogènes pour effectuer la sélection [et maintenir une] nécessaire unité de doctrine ». La réalisation de l'inventaire relèverait du département ou de la commune qui sont des niveaux de «gestion de proximité».

On voit bien que ce dispositif fonctionnel et hiérarchique, plaçant l'État au niveau de la science, de la doctrine et de la méthode, le met à l'abri des conséquences désagréables des effets politiques et sociaux de leur application. Ce qui est transféré aux collectivités territoriales, c'est la responsabilité d'avoir à gérer l'écart entre les principes et leur mise en œuvre. Car la nécessaire distance entre la loi générale et les intérêts particuliers n'est pas réduite par la proximité géographique. Au contraire, les principes que sont la définition du patrimoine national et des critères présidant à sa préservation risquent de céder le pas devant la pression des intérêts particuliers des propriétaires privés et des collectivités territoriales ou locales.

La rhétorique argumentative est cependant séduisante. Il s'agirait ni plus ni moins de «passer d'une politique étatique à une politique nationale du patrimoine». Ce qui serait recherché, c'est «l'appropriation collective du patrimoine par les habitants d'une commune, d'un département, d'une région ». Pour cela, il faut «que les décisions soient prises au plus près des citoyens, ce qui induit un rôle accru des collectivités territoriales et des propriétaires privés (pas seulement en tant que propriétaires, mais aussi comme citoyens sensibilisés et relais d'une politique patrimoniale) ». La proximité n'est plus alors simplement celle du territoire, mais la fusion dans une volonté partagée du bien commun et de l'intérêt particulier. Les monuments peuvent être nationaux sans être propriété de l'État4.

\section{Le transfert de propriété du patrimoine monumental national : proximus versus longissimus}

Le 19 novembre 2003 au matin, la moitié des Alsaciens (les habitants du BasRhin) se sont réveillés en apprenant qu’ils allaient devenir propriétaires d'un

4. Plan national pour le patrimoine : « 56 propositions pour passer d’une politique étatique à une politique nationale dotée des moyens nécessaires», Direction de l'architecture et du patrimoine, avril 2003. 
des plus grands monuments historiques nationaux de France situé sur leur territoire : le château du Haut-Kœnigsbourg. Il ne s'en est guère trouvé pour s'étonner qu'en cette période de débat sur la démocratie de proximité, ils n’aient jamais été consultés sur la question. Il est probable que dans leur inconscient - qui a eu le privilège d'être psychanalysé ${ }^{5}$ - ils se soient toujours sentis propriétaires d'un monument dont, en d'autres temps, ils avaient financé la moitié de la restauration. Sans doute aussi sont-ils fiers que leur président (du conseil général) soit le seul à s'être déclaré spontanément disposé à accepter le don proposé, alors que «nous ne sentons pas une réelle demande des collectivités territoriales dans ce domaine; elles subodorent que cela va leur couter cher ", comme le remarque la commission Bady. Il faut dire que le château est, parmi les 130 édifices du Centre national des monuments historiques, un des rares à produire des bénéfices grâce aux 500000 visiteurs annuels. Une telle aubaine vaut bien que l'on oublie que cet argent contribue à financer la conservation de plus d'une centaine d'autres témoins de l'histoire nationale, moins lucratifs mais plus éloignés. Mais «les Alsaciens» sont-ils prêts à en payer le prix symbolique?

Une commission présidée par René Rémond a remis au ministre de la Culture, le 17 novembre 2003, un rapport sur le transfert aux collectivités locales de monuments historiques appartenant aujourd'hui à l'État ${ }^{6}$. Il identifie «un certain nombre de critères justifiant le maintien de certains édifices à l'État : appartenance à la mémoire de la nation, rayonnement européen ou international, conservation particulièrement délicate». Passant les quelque 300 monuments au crible de ces critères, il considère "souhaitable» ou "possible» le transfert de plus de la moitié d'entre eux, sans débat notable au sein de la commission. Un seul canard boiteux : le Haut-Kœnigsbourg. «Ce monument n'entre manifestement pas dans la rubrique des résidences des anciennes dynasties françaises et appartient prioritairement à la mémoire de l'Alsace, mais il est aussi le témoin des vicissitudes qui affectèrent les relations entre la France et l'Allemagne. Il a été l'objet d'une disposition du traité de Versailles; l'État a consenti pour ce monument d'importants efforts. De surcroit, il présente pour l'histoire des conceptions en matière de restauration un intérêt comparable à celui de Pierrefonds ou Carcassonne, que la Commission a proposé de laisser dans le domaine de l'État." La commission, partagée, s'en remet alors prudemment à l'avis des pouvoirs publics.

Dans la nuit du 16 au 17 février 1995, une forte bourrasque de vent d'ouest a fait tomber la statue de l'aigle qui dominait le donjon du château depuis sa restauration en 1908, délivrant de ses entrailles un parchemin que l’on place

5. F. Hoffet, 1951 [et rééditions], Psychanalyse de l'Alsace, Alsatia Éditions.

6. Rapport de la Commission présidée par René Rémond au ministre de la Culture, 17 novembre 2003. 
habituellement dans la première pierre d'une construction7. Il y est notamment écrit : « [L’aigle] étend ses ailes sur le château reconstruit et sur le Reichsland qui s'étend à ses pieds. C'est un emblème qui dans le château reconstruit, avec la grâce de Dieu, doit être dressé aux yeux des peuples pour des siècles. C'est l'élément essentiel qui couronne une œuvre qui se dressera encore dans l'avenir et témoignera de la disponibilité du peuple pour aider son seigneur impérial dans la reconstruction d'un monument de la puissance et de la majesté allemande.» Or c'est la ville de Sélestat qui avait fait don des ruines de ce château - qui l'encombraient - à l'empereur Guillaume II, et la moitié de la somme nécessaire à la restauration a été fournie par l'Alsace-Lorraine, en échange de contreparties politiques il est vrai. On imagine alors les frissons des Alsaciens d'aujourd'hui que l'empereur remerciait par avance d'avoir contribué à signifier pour des siècles la légitimité dynastique des Hohenzollern dans la continuité des Habsbourg, et avant eux des Hohenstaufen, propriétaires successifs du château.

En 1918, l'Alsace et le Haut-Kœnigsbourg sont rendus à la France. Même si ce n'est pas toujours sans ambigüité, l'Alsace assume son passé et n'a pas d'état d'âme sur son appartenance présente. Son histoire allemande, comme sa langue germanique, fait partie de son identité française. On peut toujours considérer son annexion entre 1871 et 1918 , ainsi que son occupation entre 1940 et 1945 , comme des «vicissitudes» de l'histoire. Il est plus difficile de renvoyer "prioritairement» ces périodes à la seule «mémoire de l'Alsace». Ou bien alors, pour justifier le transfert de propriété d'un monument national de l'État à la collectivité territoriale, on ampute la mémoire nationale d'une partie de son histoire qui la dérange. On voit bien que la localisation de sa gestion repose sur une transformation de la signification du bien patrimonial. On comprend mal, alors, les motivations des élus de cette région à vouloir s'approprier les deux faces du symbole. L'idée de patrimoine de proximité contient la même contradiction logique que celle de télévision de proximité (outil pour voir de loin ce qui est proche). Car paradoxalement, le geste qui rapproche le patrimoine national du territoire l'éloigne d'autant de ce qui lui a donné sa signification. Le monument est comme le signe de Saussure : sa forme et son sens sont inséparables, comme les deux côtés d'une feuille de papier. Transformer l'une c'est modifier l'autre. Mais en même temps, leur rapport n'est pas analogique : la valeur symbolique n'est pas une caractéristique de l'objet. Le monument ne devient patrimoine que par décision. Le «proximoine» est de ce fait une illusion. Gérer la mémoire collective suppose de la distance.

7. M. Fuchs, juin 1995, "L'envol de l'aigle», Annuaire des amis de la Bibliothèque humaniste de Sélestat. 


\section{Bibliographie}

CHOAY F., 1996, L'allégorie du patrimoine, Paris, Le Seuil.

HARTOG F., 2003, Régimes d'historicité. Présentisme et expérience du temps, Paris, Le Seuil.

Haut-Kœnigsbourg, Connaissance des Arts, publication de la Caisse nationale des monuments historiques et des sites, 1996. 